\title{
Viewpoint: Animal-Unit Equivalents Cannot Be Meaning- fully Weighted by Indices of Dietary Overlap
}

\section{DAVID L. SCARNECCHIA}

In fact, even if animal-unit equivalents (AUE) could be meaningfully weighted by dietary differences, their rigorous use in systems analysis demands that they should not be weighted. First, the "cannot."

Animal-unit equivalents are simple values expressing the demand of animals in animal-units; they are not substitution ratios. The example of Hobbs and Carpenter (1986) in which 7 deer have the same "impact" as 1 cow shows a substitution ratio, not an animal-unit equivalent. Calculation of substitution ratios is a modeling process, equating specific variables and involving specific objectives (Scarnecchia 1985). Hobbs and Carpenter (1986) give no example of how to weight animal-unit-equivalents (or substitution ratios) based on dietary differences. If we use the method of Flinders and Conde (1979), the weighing is done by use of a relative index of dietary overlap between two animal species. In their example, sheep and cattle have a $35 \%$ diet overlap, and rather than having 1 cow "=" 5 sheep, 1 cow " $=" 5 / 0.35=14.3$ sheep.

The problems of this approach are many and compounded, but the major problems are: (1) It is unclear in terms of what variable(s) 14.3 sheep "equals" 1 cow. The approach confuses animal-unit equivalents with species substitution ratios, is unspecific as to the variable(s) involved, and amounts to modeling objective-specific substitution ratios without specific objectives (Scarnecchia 1985). (2) An index of similarity used (Kulczinski's Index was used by Flinders and Conde) is a relative index used to compare dietary overlaps among a group of pairs of species; most indices have no physical interpretation and although some may be treated statistically, all have complex implicit units, and are generally not useful in subsequent mathematical calculations. In other words, the number of sheep to be substituted for a cow can be divided by a dietary overlap index in the sense that you can always divide one number by a nother, but it cannot be meaningfully divided because the relative dietary overlap index makes the quotient dimensionally complex and its meaning unclear. In what sense, i.e., in terms of what variable does 14.3 sheep equal 1 cow? There is no answer.

Now the "should not." An animal-unit should be a unit of animal. That sounds simple enough, but requires that AUE's be functions of variables describing animal characteristics (requirements for maintenance, lactation, etc.) and not variables describing animal-pasture or other animal-environment interactions. It

Author is assistant professor, Department of Forestry and Range Management, Washington State University, Pullman, Wash. 99164-6410. also requires animal-units be units of animal demand, not units of animal intake (Scarnecchia 1985; Scarnecchia and Gaskins, [in press]), because intake is clearly a function of many animal-pasture and animal-environment interactions. An animal-unit cannot be both a unit of demand and a unit of intake. Defining animal-units as units of intake, and AUE's as functions of intake has clear disadvantages (Scarnecchia 1985), not the least of which is that intake is difficult to measure, model or predict. More importantly, any combination of animal-unit-equivalents with interactive variables (most notably, intake) produces confounding of AUE's and derived stocking rates, etc., with those interactive variables. Relationships such as stocking rate vs. intake are difficult to interpret because they are confounded. Our thinking should be integrative and synergetic; the basic variables (such as AUE's) used in our analyses minimally confounded to be useful in systems analysis.

At best, an animal-unit can cleanly be unit of either (1) energy demand, (2) energy intake (3) dry matter intake or (4) dry matter forage supply (Scarnecchia and Gaskins [in press]). To have it vaguely be a unit of all of these variables is not good science, and not the basis of good management. Animal-unit-equivalents should express the demand of animals in animal-units; they should not involve diet quality, diet selection or other complex interactive processes. If systematically limited in this way, (Scarnecchia 1985; Scarnecchia and Gaskins, [in press]), the animal-unit and animalunit-equivalent concepts can be used in quantifying animal demand in supply/demand analyses of range-livestock systems, and in calculations of animal-unit-months, animal-unit-days and stocking variables.

\section{Literature Cited}

Flinders, Jerran T., and Linda Conde. 1979. An alternative method of calculating animal-unit equivalents helps ranchers and wildlife (abstract). Paper presented at the 33rd Annual Meeting, Soc. for Range Manage., San Diego, Calif., Feb. 11-14.

Hobbs, N. Thompson and Len H. Carpenter. 1986. Viewpoint: animal-unit equivalents should be weighted by dietary differences. J. Range Manage. 39:470.

Scarnecchia, David. L. 1985. The animal-unit and animal-unit equivalent concepts in range science. J. Range Manage. 38:346-349.

Scarnechia, David L., and Charles T. Gaskins. Modeling animal-unit equivalents for beef cattle. (In press). 